\title{
Numerical Simulation of Hydraulic Transport of Sand-Water Mixtures in Pipelines
}

\author{
Changhee Kim ${ }^{1}$, Cheolheui Han ${ }^{2 *}$ \\ ${ }^{1}$ Hyundai Engineering \& Construction Company, Seoul, South Korea \\ ${ }^{2}$ Department of Aeronautical and Mechanical Design, Korea National University of Transportation, Chungju, South Korea \\ Email: "chhan@ut.ac.kr,kimch@hdec.co.kr
}

Received August 13, 2013; revised September 13, 2013; accepted September 20, 2013

Copyright (C) 2013 Changhee Kim, Cheolheui Han. This is an open access article distributed under the Creative Commons Attribution License, which permits unrestricted use, distribution, and reproduction in any medium, provided the original work is properly cited.

\begin{abstract}
The development of empirical model for the hydraulic transport of sand-water mixtures is important for the design of economical solid-liquid transportation system in chemical and waste-disposal industries. The hydraulic transport characteristics of sand-water mixtures in circular pipelines are numerically investigated by using the FLUENT commercial software. Eulerian granular multiphase (EGM) model with the k-e turbulent model is used for the computation. Present method is validated by the computed values with the measured data. The effect of the concentration and pipe sizes on the relative solid effect is numerically investigated. It is found that the effect of the volumetric delivered concentration on both hydraulic gradient and solid effect increases as the Reynolds number decreases. When the Reynolds number is small, the increase in the volumetric delivered concentration has an effect of decreasing the hydraulic gradient whereas the solid effect increases with the volumetric delivered concentration stepping up. The effect of the pipe diameter is not the critical parameter for deciding the values of the relative solid effect in the sand-water mixture transportation.
\end{abstract}

Keywords: Computational Fluid Dynamics; Hydraulic Transport; Solid Effect; Sand-Water Mixture

\section{Introduction}

Solid-liquid flow in pipelines has been a popular mode of transportation in chemical and waste-disposal industries. Circular pipes are normally used for long distance transportation of variety of materials in bulk quantities [1]. Both time and pumping power required for hydraulically transporting huge amounts of soils in a state of slurry (sand-water mixture) are crucial factors for successful dredging and reclaiming projects [2]. Thus, the economical hydraulic transportation of solids to a long distance has been attempted by using several methods $[3,4]$.

During the several past decades, the complex computational scheme describing the microscopic processes in the solid-liquid flow and the computing costs has been a drawback to the development and use of commercial softwares. Ling et al. [5] investigated the double slurry flow in the pipe for the fully turbulent flow using Eulerian granular multiphase (EGM) model. Al Araby et al. [6] performed a numerical study on the single phase combined free and force convection in the entrance region of a horizontal pipe with its wall temperature fixed

${ }^{*}$ Corresponding author. as a constant value. Lin and Ebadian [7] used a simplified three-dimensional algebraic slip mixture (ASM) model [8] and the RNC k-e turbulent model [9] while focusing on the developing process of volume of fraction and density distributions, mean velocity profiles.

Kim et al. [10] experimentally investigated the hydraulic transport characteristics of sand-water mixtures in circular and square pipelines by changing the Reynolds number and volumetric delivered concentration.

In the present paper, the numerical computation results are presented by first validating the numerical results with the measured data. The present study is also mainly focused on the heterogeneous and homogeneous flow regimes. The effect of the concentration and the geometrical changes on the relative solid effect will be discussed.

\section{Numerical Method}

\subsection{Governing Equations}

The continuity equation for the mixture is

$$
\frac{\partial}{\partial t}\left(\alpha_{q} \rho_{q}\right)+\nabla \cdot\left(\alpha_{q} \rho_{q} v_{q}\right)=\sum_{p=1}^{n} \dot{m}_{p q}
$$


where $\alpha_{q}$ denotes the volume fraction of single material $q$ among the materials that constitutes the mixture, $\rho_{q}$ is the density of a material $q, \dot{m}_{p q} \dot{m}_{p q}$ is the mass flow rate transferred from the $p^{\text {th }}$ material to the $q^{\text {th }}$ material.

The momentum equation for the single material $q$ can be expressed as

$$
\begin{aligned}
& \frac{\partial}{\partial t}\left(\alpha_{q} \rho_{q} \boldsymbol{v}_{q}\right)+\nabla \cdot\left(\alpha_{q} \rho_{q} \boldsymbol{v}_{q} \boldsymbol{v}_{q}\right) \\
& =-\alpha_{q} \nabla p+\nabla \cdot \overline{\overline{\tau_{q}}}+\sum_{p=1}^{n}\left(\boldsymbol{R}_{p q}+\dot{m}_{p q} \boldsymbol{v}_{p q}\right) \\
& \quad+\alpha_{q} \rho_{q}\left(\boldsymbol{F}_{q}+\boldsymbol{F}_{l i f t, q}+\boldsymbol{F}_{v m, q}\right)
\end{aligned}
$$

where the stress-strain tensor of a material $q, \bar{\tau}$, is

$$
\bar{\tau}=\alpha_{q} \mu_{q}\left(\nabla \boldsymbol{v}_{q}+\nabla \boldsymbol{v}_{q}\right)+\alpha_{q}\left(\lambda_{q}-\frac{2}{3} \mu_{q}\right) \nabla \cdot \boldsymbol{v}_{q} \overline{\bar{I}}
$$

where $\mu_{q}$ and $\lambda_{q}$ is the shear and bulk viscosity of the material $q$, respectively. $\boldsymbol{F}_{q}$ denotes the external force, $\boldsymbol{F}_{l i f t, q}$ is the lift force and $\boldsymbol{F}_{v m, q}$ is the virtual mass force. $\boldsymbol{R}_{p q}$ represents the interaction among the materials, $p$ is the static pressure.

$$
\begin{aligned}
& \frac{\partial}{\partial t}\left(\alpha_{q} \rho_{q} \boldsymbol{v}_{q}\right)+\nabla \cdot\left(\alpha_{q} \rho_{q} \boldsymbol{v}_{q} \boldsymbol{v}_{q}\right) \\
& =-\alpha_{q} \nabla p+\nabla \cdot \overline{\tau_{q}}+\alpha_{q} \rho_{q} \boldsymbol{g}+\alpha_{q} \rho_{q}\left(\boldsymbol{F}_{q}+\boldsymbol{F}_{l i f t, q}+\boldsymbol{F}_{v m, q}\right) \\
& \quad+\sum_{p=1}^{n}\left(K_{p q}\left(\boldsymbol{v}_{p}-\boldsymbol{v}_{q}\right)+\dot{m}_{p q} \boldsymbol{v}_{p q}\right) \\
& \frac{\partial}{\partial t}\left(\alpha_{s} \rho_{s} \boldsymbol{v}_{s}\right)+\nabla \cdot\left(\alpha_{s} \rho_{s} \boldsymbol{v}_{s} \boldsymbol{v}_{s}\right) \\
& =-\alpha_{s} \nabla p-\nabla p_{s}+\nabla \cdot \bar{\tau}_{s}+\alpha_{s} \rho_{s} \boldsymbol{g} \\
& +\alpha_{s} \rho_{s}\left(\boldsymbol{F}_{s}+\boldsymbol{F}_{l i f t, s}+\boldsymbol{F}_{v m, s}\right)+\sum_{l=1}^{n}\left(K_{l s}\left(\boldsymbol{v}_{l}-\boldsymbol{v}_{s}\right)+\dot{m}_{l s} \boldsymbol{v}_{l s}\right)
\end{aligned}
$$

where $K_{l s}, K_{s l}$ are the coefficients that represent the momentum transfer between the fluid and the soild, and can be denoted as follows

$$
\begin{aligned}
& K_{l s}= \\
& \frac{3\left(1+e_{l s}\right)\left(\frac{\pi}{2}+C_{f r l s} \frac{\pi^{2}}{8}\right) \alpha_{s} \rho_{s} \alpha_{l} \rho_{l}\left(d_{l}+d_{s}\right)^{2} g_{o, l s}}{2 \pi\left(\rho_{l} d_{l}^{3}+\rho_{s} d_{s}^{3}\right)}\left|\boldsymbol{v}_{l}-\boldsymbol{v}_{s}\right|
\end{aligned}
$$

The shear stress of the solid includes the shear viscosity $\left(\mu_{s}\right)$ due to the collision and transfer among the particles and the bulk viscosity $\left(\lambda_{s}\right)$, and can be written as follows:

$$
\mu_{s}=\mu_{s, c o l}+\mu_{s, k i n}+\mu_{s, f r}
$$

where $\mu_{s, c o l}$ is the component due to collision, $\mu_{s, k i n}$ is the component due to motion, and $\mu_{s, f r}$ is the fric- tional component. In the present study, the k-e turbulence model is used for the eddy viscosity modeling.

\subsection{Boundary Conditions}

In the present study, the mean velocity inlet boundary condition and pressure outlet boundary condition are imposed on the inlet and the outlet of the pipeline, respectively. The turbulence intensity level is set to $4 \%$ for the intermediate velocities in the sand-water mixture flows. The pipe length $\mathrm{L}$ was set to 100D. The density of water is $998.2 \mathrm{~kg} / \mathrm{m}^{3}$. The specific gravity $(\mathrm{Ss})$ and diameter $\left(\mathrm{d}_{50}\right)$ of Jumunsin sands are 2.65 and $0.54 \mathrm{~mm}$, respectively [10]. Water temperature is set to $20^{\circ} \mathrm{C}$.

A multi-block unstructured, non-uniform grid system with hexahedral elements is used to discretize the computation domain. Using the symmetry boundary condition, the computational domain is set to the half of the physical domain. Heat transfer is neglected with the steady state slurry flow assumption. The second-order upwind and central difference are selected as the discretization schemes of both convection and diffusion terms, respectively. With the SIMPLEC algorithm for the coupling between the velocity and pressure, under-relaxation technique is applied in the dependent variables.

\section{Results and Discussion}

In order to validate the present method computed results are compared with measured data. Figure 1 shows the velocity profiles from the bottom of the circular pipe to the top. Present results are compared with the measured data in [11]. The diameters of the pipe and the sand in the experiment are $51.5 \mathrm{~mm}$ and $0.165 \mathrm{~mm}$, respectively. The volumetric delivered concentration $\left(C_{v d}\right)$ is $8.4 \%$. Though the present computation slightly over-predicts at the center and under-predicts at the point where the flow velocity has the maximum value, the present results agree with the measured data.

Figure 2 shows the change of the hydraulic gradient due to the changes in the Reynolds number and the volumetric delivered concentration $\left(C_{v d}\right)$. The amount of solids delivered inside the pipeline is represented by the volumetric delivered concentration $\left(C_{v d}\right)$ that is calculated as the ratio between solid and slurry flow rates $\left(Q_{s} / Q_{m}\right)$. The Reynolds number is defined as $V_{m} D_{h} \rho_{w} / \mu_{w}$, where $D_{h}$ and $\mu_{w}$ represent the hydraulic diameter of the pipes and the viscosity of the water, respectively. Mean velocity $\left(V_{m}\right)$ is a bulk velocity of the mixture defined as the volumetric flow rate of a matter passing through a pipeline, $Q_{m}$, divided by the cross-sectional area, $A$.

$$
V_{m}=\frac{Q_{m}}{A}=\frac{4 Q_{m}}{D^{2}}
$$

where $Q_{m}$ equals the sum of $Q_{s}$ and $Q_{f}$ that represent the 


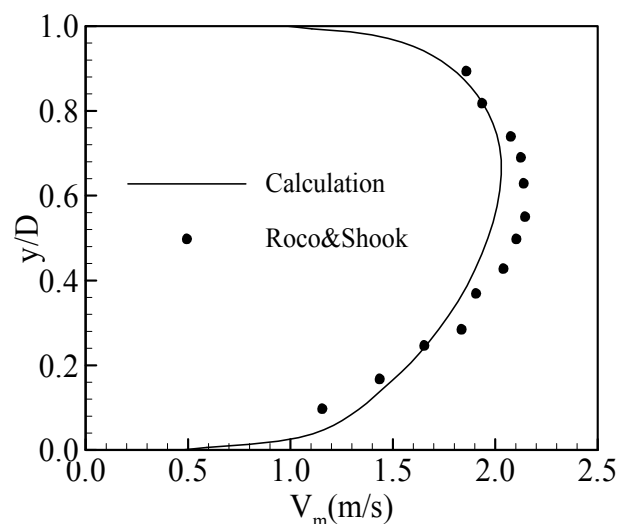

Figure 1. Comparison of velocity profile at $C_{v d}=8.4 \%, d=$ $0.165 \mathrm{~mm}$ and $D=51.5 \mathrm{~mm}$.

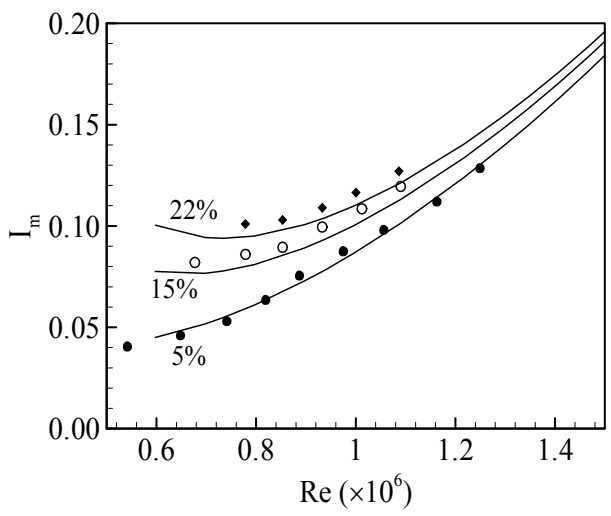

Figure 2. Concentration $\left(C_{v d}\right)$ effect to hydraulic gradient $\left(I_{m}\right)$ at $d=0.54 \mathrm{~mm}$ and $D=200 \mathrm{~mm}$.

flow rates of the solid particles and the fluid, respectively. In this paper, the definition of the Reynolds number is also used for water and slurry flows together. Present results are compared with the measured data [2]. The diameters of the pipe and the sand in the experiment are $150 \mathrm{~mm}$ and $0.54 \mathrm{~mm}$, respectively. It can be seen from the figure that present results agree well with the measured data when the Reynolds are within the range from $0.7 \times 10^{6}$ to $1.3 \times 10^{6}$.

Figure 3 shows the relation of the volumetric delivered concentration with the hydraulic gradient $\left(I_{m}\right)$ in the given Reynolds number. The energy loss due to friction can be assessed by using hydraulic gradient $\left(I_{m}\right)$ that means the pressure gradient for a mixture expressed in height of water per length of line.

$$
I_{m}=\frac{\Delta p}{\rho_{f} g L}
$$

As shown in Figure 3, the effect of the volumetric delivered concentration on hydraulic gradient increases as the Reynolds number decreases. When the Reynolds number is small, the increase in the volumetric delivered concentration has an effect of decreasing the hydraulic gradient.

Figure 4 shows the relation of the volumetric delivered concentration with solid effect $\left(I_{m}-I_{w}\right)$ in the given Reynolds number. In evaluating additional friction loss due to the solid particles, solid effect represented by $\left(I_{m}-I_{w}\right)$ is used. $I_{w}$ means the friction gradient for water alone at the flow rate equal to the mixture flow rate $\left(Q_{m}\right)$. Figure 4 shows that the effect of the volumetric delivered concentration on solid effect increases as the Reynolds number decreases. When the Reynolds number is small, the solid effect increases with the volumetric delivered concentration stepping up. Thus, it can be said that, when the flow speed is low, the friction among the solid particles becomes a dominant factor, which results in the increase in the energy loss due to friction. When the flow speed is high, the solid particle distributions become uniform and the solid particles flow through the pipe with less probability of settling on the bed. Thus, the friction between the solid particles with the wall or deposited solid particles becomes small.

Figure 5 shows the effect of the solid particle size on the relative solid effect as a function of the Reynolds number $\left(V_{m} D_{h} \rho_{w} / \mu_{w}\right)$. It can be seen from the figures that the large size solid particles has the large relative

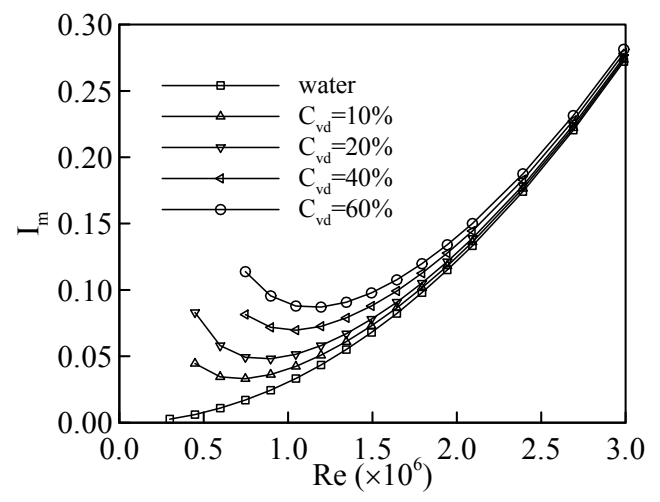

Figure 3. Concentration $\left(C_{v d}\right)$ effect on both hydraulic gradient $\left(I_{m}\right)$ hydraulic gradient $\left(I_{m}\right)$.

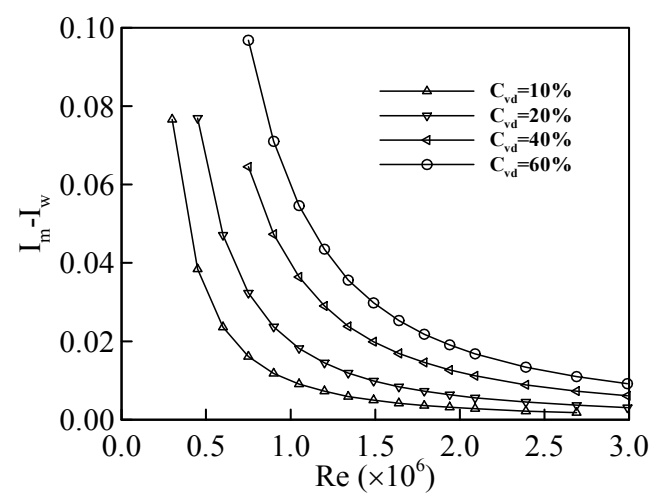

Figure 4. Concentration $\left(C_{v d}\right)$ effect on both hydraulic gradient $\left(I_{m}\right)$ and solid effect $\left(I_{m}-I_{w}\right)$. 


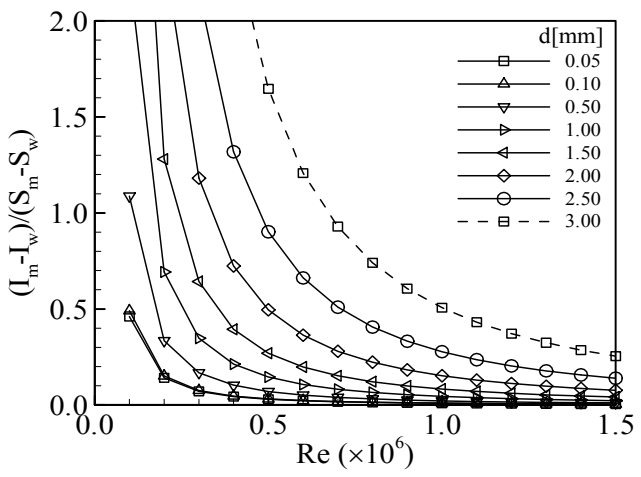

(a)

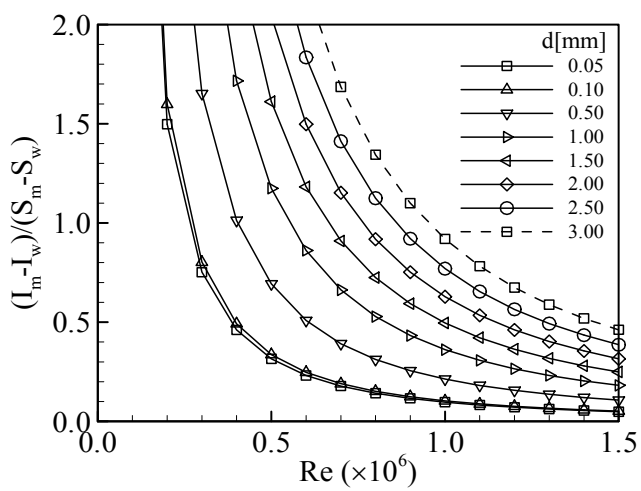

(b)

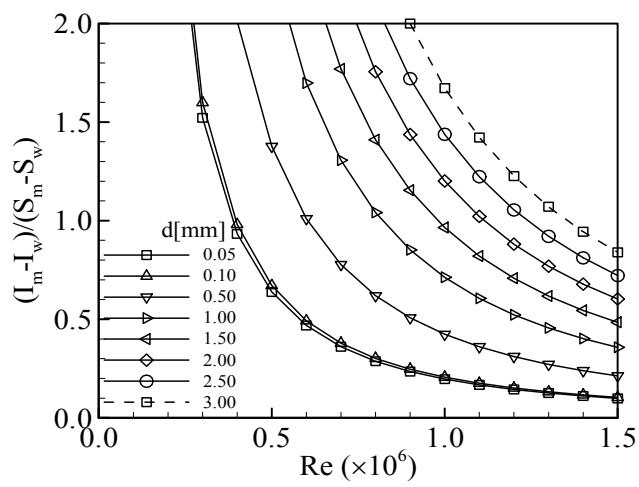

(c)

Figure 5. Correlation of data with relative solid effect. (a) $D$ $=100 \mathrm{~mm}$; (b) $D=400 \mathrm{~mm}$; (c) $D=600 \mathrm{~mm}$.

solid effect. However, when the solid particle size is very small $(d<0.1 \mathrm{~mm})$, the particle size does not affect the value of the relative solid effect. Wilson et al. [12] found the following relationship.

$$
\frac{I_{m}-I_{w}}{S_{m}-S_{w}} \approx 0.22\left(\frac{V_{m}}{V_{50}}\right)^{-M}
$$

where 0.22 represents the value of the relative solid effects when $V_{m}=V_{50}$. The magnitude of $M$ is a function of normal stress of solids (granular pressure). In Equation (9), $S_{w}=1$ because the working fluid is water. Following Equation (9), we can represent the relative solid effect as a function of the Reynolds number.

$$
\frac{\left(I_{m}-I_{w}\right)}{\left(S_{m}-S_{w}\right)}=A \cdot \mathrm{Re}^{-M}
$$

According to the estimation of Clift et al. [13], the value of $M$ is set to 1.7. It can be said from the figures that the relative solid effect is affected more by the particle size than the pipe diameter.

The effect of the pipe diameter on the relative solid effect can be represented as follows [14]:

$$
\frac{\left(I_{m}-I_{w}\right)}{\left(S_{m}-S_{w}\right)}=A \cdot\left(\frac{V_{m}}{U_{u}^{\prime}}\right)^{-M}
$$

Table 1 and Figure 6 show the effect of non-dimensionalized particle size effect on the relative solid effect as a function of the Reynolds number. As shown on Table 1 , the non-dimensionalized particle size is set to 0.0025 for the investigated 5 cases. The value of $M$ is also set to 1.7 , then the value of $A$ is obtained by computing the relative solid effect with the change in the mean velocity. It can be seen from the Table 1 and Figure 6, that, even though the non-dimensionalized particle size has the same value, the relative solid effect has different values. In case the diameters of both pipe and solid particle are large, the values of the relative solid effect are larger than the other cases. Thus, it can be said from the results in Figure 6 that the size of the pipe diameter compared to the solid particle size is not the dominant factor. The particle size itself is a critical parameter to the performance of the slurry transportation system.

\section{Conclusions}

In this paper, the effect of the concentration, and both particle and pipe sizes on the relative solid effect is numerically investigated using Eulerian granular multiphase (EGM) model and k-e turbulent model. Present method is validated by the computed values with the measured data.

It is found that the effect of the volumetric delivered concentration on both hydraulic gradient and solid effect increases as the Reynolds number decreases. When the Reynolds number is small, the increase in the volumetric delivered concentration has an effect of decreasing the

Table 1. Correlation coefficient at $d / D=0.0025$.

\begin{tabular}{cccccc}
\hline & $\boldsymbol{D}(\mathbf{m m})$ & $\boldsymbol{d}(\mathbf{m m})$ & $\boldsymbol{d} / \boldsymbol{D}$ & $\boldsymbol{A}$ & $\boldsymbol{M}$ \\
\hline Case 1 & 200 & 0.5 & 0.0025 & 0.1288 & 1.7 \\
Case 2 & 400 & 1 & 0.0025 & 0.2217 & 1.7 \\
Case 3 & 600 & 1.5 & 0.0025 & 0.2883 & 1.7 \\
Case 4 & 800 & 2 & 0.0025 & 0.3535 & 1.7 \\
Case 5 & 1000 & 2.5 & 0.0025 & 0.4150 & 1.7 \\
\hline
\end{tabular}




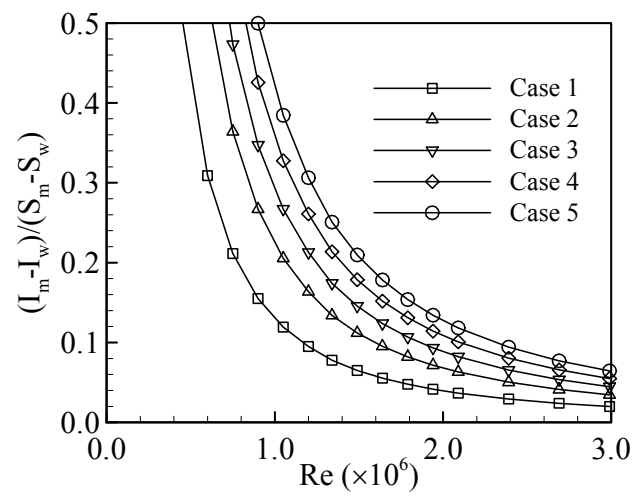

Figure 6. Correlation of data with relative solid effect $\left(I_{m}-\right.$ $\left.I_{w}\right) /\left(S_{m}-S_{w}\right)$.

hydraulic gradient whereas the solid effect increases with the volumetric delivered concentration stepping up.

The effect of the particle size and the pipe diameter on the relative solid effect is calculated and correlated using the previously developed simple model. It is found that the effect of the pipe diameter or the relative size of the particle size with regard to the pipe diameter is not the critical parameter for deciding the values of the relative solid effect in the sand-water mixture transportation. The particle size itself is shown to be the most important parameter when the concentration is fixed.

In future, the effect of the particle size on the deposition limit velocity will be discussed.

\section{REFERENCES}

[1] D. R. Kaushal and Y. Tomita, "Comparative Study of Pressure Drop in Multisized Particulate Slurry Flow through Pipe and Rectangular Duct," International Journal of Multiphase Flow, Vol. 29, No. 9, 2003, pp. 14731487. http://dx.doi.org/10.1016/S0301-9322(03)00125-3

[2] V. Matousek, "Flow Mechanism of Sand-Water Mixtures in Pipelines," Delft University Press, Delft, 1997.

[3] M. E. Charles, "Transport of Solids by Pipeline," Proceedings of Hydrotransport 1, BHRA Fluid Engineering, Cranfield, England, Paper A3.

[4] G. K. Patterson, J. L. Zakin and J. M. Redriguez, "Drag Reduction: Polymer Solutions, Soap Solution and Particle Suspensions in Pipe Flow," Industrial Engineering of Chemistry, Vol. 61, No. 1, 1969, pp. 22-35. http://dx.doi.org/10.1021/ie50709a005

[5] J. Ling, C. X. Lin and M. A. Ebadian, "Numerical Investigation of Double-Species Slurry Flow in a Straight Pipe Entrance," ASME 2002 International Mechanical Engineering Congress and Exposition, Heat Transfer, Vol. 4, New Orleans, 17-22 November 2002, pp. 145-154.

[6] M. A. Al Araby, M. K. Shaban, A. S. Salem and M. M. Mahmoud, "Laminar Combined Free \& Forced Convection Heat Transfer in the Entry Length of a Horizontal Pipe," Proceedings of the ASME Heat Transfer/Fluid Engineering Summer Conference, HT/FED 2004, Vol. 1, Charlotte, 11-15 July 2004, pp. 541-551.

[7] C. X. Lin and M. A. Ebadian, "A Numerical Study of Developing Slurry Flow in the Entrance Region of a Horizontal Pipe," Computers and Fluids, Vol. 37, No. 8, 2008, pp. 965-974. http://dx.doi.org/10.1016/j.compfluid.2007.10.008

[8] M. Manninen, V. Taivassalo and S. Kallio, "On the Mixture Model for Multiphase Flow," Technical Research Center of Finland, Espoo, VIT Publications 288, 1996.

[9] B. E. Launder and D. B. Spalding, "The Numerical Computation of Turbulent Flows," Computer Method in Applied Mechanical Engineering, Vol. 3, No. 2, 1974, pp. 269-289.

http://dx.doi.org/10.1016/0045-7825(74)90029-2

[10] C. H. Kim, M. S. Lee and C. Han, "Hydraulic Transport of Sand-Water Mixtures in Pipelines, Part I Experiments," Journal of Mechanical Science and Technology, Vol. 22, No. 12, 2008, pp. 2534-2541. http://dx.doi.org/10.1007/s12206-008-0811-0

[11] M. V. Roco and C. A. Shook, "Modeling of Slurry Flow: The Effect of Particle Size," Canadian Journal of Chemical Engineering, Vol. 61, No. 4, 1983, pp. 494-503. http://dx.doi.org/10.1002/cjce.5450610402

[12] K. C. Wilson, "Influence of Particle Properties on Solid Effects," Proceedings of the 10th International Kol. Massenguttransport Druch Rohrleitungen, University. GH Paderborn, Meschede, Germany, 1992.

[13] R. Clift, K. C. Wilson, G. R. Addie and M. R. Cartens, "A Mechanistically-Based Method for Scaling Pipeline Tests for Settling Slurries," Proceedings Hydrotransport 8. BHRA Fluid Engineering, Cranfield, 1982, pp. 91-101.

[14] G. R. Addie and J. R. Hammer, "Pipeline Head Loss Test of Settling Slurries at Low Velocities," International Mechanical Engineering Congress, Perth Western, 1994, pp. 225-230. 